\title{
Who Should Manage Periprosthetic Joint Infection? The Case for a Multidisciplinary Approach
}

\author{
S. Yacovelli, J. Parvizi \\ Rothman Orthopedic Institute, Thomas Jefferson University Hospital, \\ Philadelphia, USA
}

\begin{abstract}
Periprosthetic joint infection (PJI) following total joint arthroplasty (TJA) is a complex disease state that is quite devastating to those affected. Improvement in diagnostic testing modalities and therapeutic techniques have led to significant advances in treatment for patients, but there is still a considerable gap in treatment success across providers and institutions. Where and who should be treating cases of PJI remains a debated topic. Many experts have proposed a new treatment model not dissimilar to that with which has been used to treat other complex disease states such as cancer for decades, and there is now a growing body of evidence to support such a strategy is superior. In this article, we evaluate the current body of literature on the topic and offer recommendations for the ideal treatment model for PJI: the multidisciplinary approach.
\end{abstract}

Keywords: periprosthetic joint infection, total joint arthroplasty, multidisciplinary approach to treatment.

\section{Кто должен лечить перипротезную инфекцию: необходимость мультидисциплинарного подхода}

\author{
С. Яковелли, Д. Парвизи \\ Ортопедический институт Ротмана, Университетский госпиталь Томаса Джефферсона, \\ Филадельфия, США
}

\section{Реферат}

Перипротезная инфекция (ППИ) после тотального эндопротезирования является сложным заболеванием, разрушительным для организма пациентов. Улучшение методов диагностики и лечения привело к значительному прогрессу в лечении этой патологии, но все еще существует значительный разрыв в успехе лечения среди поставщиков и учреждений. Где и кто должен лечить пациентов с ППИ остается темой дискуссии. Многие эксперты предлагают новую модель лечения, не отличающуюся от той, которая использовалась в течение десятилетий для лечения других сложных заболеваний, таких как рак, и в настоящее время появляется все больше доказательств того, что такая стратегия является лучшей. В этой статье авторы приводят анализ современной литературы по этой теме и предлагают междисциплинарный подход в качестве идеальной модели лечения ППИ.

Ключевые слова: перипротезная инфекция, тотальное эндопротезирование, междисциплинарный поход к лечению.

\section{Background}

Periprosthetic joint infection (PJI) is a devastating complication of total joint arthroplasty (TJA) that leads to significant patient morbidity and mortality. Rates of infected knees following total knee arthroplasty (TKA) have been reported at $0.92 \%$ and rates of infected hips following total hip arthroplasty (THA) have been reported at a slightly lower rate of $0.88 \%$ [1]. The number of TJA cases overall have steadily increased in the United States over the past decade, likely due in part to an aging population and the obesity epidemic. The increasing incidence of PJI, coupled with improvements in understanding and diagnostic testing, means there will be further demand on the

[D] Cite as: Yacovelli S., Parvizi J. Who Should Manage Periprosthetic Joint Infection? The Case for a Multidisciplinary Approach. Travmatologiya $i$ ortopediya Rossii [Traumatology and Orthopedics of Russia]. 2019;25(4):28-32. doi: 10.21823/2311-2905-2019-25-4-28-32.

$\triangle$ Steven Yacovelli; e-mail: Steven.yacovelli@rothmanortho.com

Received: 11.11.2019. Accepted for publication: 02.12.2019. 
health care system to adequately treat this complex complication of TJA in an appropriate and cost-effective manner.

Diagnostic testing has also greatly expanded our understanding of PJI, resulting in a need for a new definition to meet the needs of clinicians handling these cases. The Musculoskeletal Infection Society (MSIS) attempted to address these concerns and provide a uniform definition and diagnostic algorithm for PJI in 2011. The advent and widespread availability of empirically validated serum and synovial markers for PJI led to a further refined diagnostic criteria recommended by the International Consensus Meeting (ICM) by 2018 [2]. While beyond the scope of this chapter, the definition includes a number of major and minor criteria, consisting of culture growth, serum CRP, ESR, and D-Dimer, synovial fluid WBC, Leukocyte Esterase, PMN\%, histological changes, and physical examination findings. This definition, while admittedly complex, allows clinicians to identify PJI despite its heterogeneous presentation and even categorize it as acute or chronic. Such nuances of the definition itself need to be recognized as they can have a profound impact on clinical decision making and patient outcomes, and as such, are best made by orthopedic specialists with experience in treating PJI. Unfortunately, change and complexity require time and experience to adjust to. Tetreault et al. evaluated the consistency of current methods for evaluating PJI according to American Academy of Orthopaedic Surgeons (AAOS) guidelines and noted an alarming gap between expectation and reality, even amongst orthopedic-trained surgeons. The study concluded that there is still significant underdiagnosis of PJI, leading to unnecessary order of advanced diagnostic imaging, adding to medical costs and a delay in treatment $[3,4]$.

The complexity of PJI doesn't stop at its diagnosis either. Various treatment strategies must be personalized on an individualized basis, and adjusted throughout the period of care in order to offer the best outcome possible. The decision to perform a single stage vs two stage revision, which antibiotic should be used and for how long it should be used for, how to medically optimize patients preoperatively, and whether or not to progress to a salvage procedure such as arthrodesis or amputation are just a few of the clinical decisions that need to be made on a multi-disciplinary level, led by an orthopedic surgeon with experience in treating PJI $[5,6]$.

For these reasons many experts have likened the ideal model with which we should treat PJI to that of the cancer care model: The multidisciplinary approach $[7,8]$. In fact, the management of both disease states is exceedingly complex, and patient outcomes are arguably comparable between the two. PJI can be devastating, with patient mortality higher than many common forms of cancer, at 5-year rates of 25$33 \%$ following two-stage exchange procedures [7, 9]. While the intricacy of cancer cases has been recognized for a long time, it was not until recently that PJI was recognized as an entity that requires a coordinated and collaborative effort amongst a variety of medical professionals in order to deliver the best care possible [10].

\section{Multidisciplinary Approach}

The days of surgeon-centered practice are long gone. Care for patients in nearly all fields has to, and needs to continue to, involve a multidisciplinary team of medical professionals $[5,7,11]$. This involves communication throughout patient care, from diagnosis to follow-up, with contributions from numerous specialists. This is best done with establishment of hospital protocol based on guidelines specific for the treatment of PJI, under the lead of a subspecialized orthopedic surgeon with experience in treating PJI [10].

As stated previously, this begins as early as the diagnostic workup. Contributions from infectious disease (ID) specialists, microbiologists, and radiologists are necessary right from the start $[9,11]$. For example, radiologists aid in recommendation of the most appropriate imaging modality and advise which areas to biopsy based on a case by case basis. In addition, ID specialists can offer information on how long antibiotics should be stopped prior to aspiration, among other contributions. Once diagnosed, patients need to be preoperatively optimized prior to surgery if possible $[9,12,13]$. Endocrinology, hematology, and nephrology are just a few of the medicine specialists that can help to minimize surgical risk, focusing on premorbid conditions such as diabetes, preoperative anemia, and chronic kidney disease, respectively, all of which are known risk factors for reinfection. Perioperatively, collaboration between surgeons and the anesthesia team is crucial as well. Hypotensive neuraxial anesthesia, use of tranexamic acid, and antibiotic administration are a few well known considerations that should be discussed before cases. Patients should be followed closely by a multidisciplinary team following surgery as well [9]. Nurse navigators, home health care providers, and nursing home staff can communicate patient progress and monitor for complications. The list of providers and their roles goes on and on and varies depending on the patient and care environment. Surgeons should be aware of the team members at their disposal and efforts should be made to establish a protocol to facilitate this process [5].

The superiority of multidisciplinary care for patients with osteoarthritis undergoing primary joint replacement has been well documented and routinely practiced for years $[7,14]$. Surgeons, anesthesiolo- 
gists, social workers, and physical therapists all play a role in the care of patients before and after surgery. Establishing an interdisciplinary care team to manage a patient together at the same facility has been shown to decrease length of stay and improve functional outcomes [14]. This is likely due to the implementation of customized treatment plans and greater comorbidity management. ${ }^{7}$ Knowing this, it can be deduced that a similar model may prove to be even more effective for the treatment of the most difficult complication of total joint arthroplasty; PJI. There is evidence in the literature to support this claim as well $[11,15]$. Ntalos et al. studied the effect of establishing a weekly multidisciplinary infection conference consisting of orthopedic surgeon, pathologists, microbiologists, and radiologists and found that it led to significant differences in treatment plans. Prior to this the hospital was using a single-discipline approach with trauma surgeons managing care and antibiotic choice. Furthermore, this study also found that by implementing multidisciplinary conferences for PJI cases, length of stay was decreased, particularly in those diagnosed with chronic PJI. This may lead to improved quality of life and lower hospital costs as well [8].

\section{Specialized Centers and Case Volume}

Access to a multidisciplinary group of healthcare providers is often difficult, and high-volume, specialized treatment centers are often best equipped to provide such care $[6,16,17]$. For this reason, treatment with a multidisciplinary approach and treatment at a high-volume tertiary care center are closely intertwined [5]. Specialized treatment centers have long reported better outcomes after primary TJA. (Bannister) In addition, fellowship-trained orthopedic surgeons who treat a high-volume of patients are better equipped to handle complex cases, and have likely developed a more refined surgical technique, established muscle memory, high attention, and faster recall [17]. Both hospital volume and surgeon volume are associated with improved clinical outcomes including decreased morbidity, mortality, and length of stay [16]. Complication rates after primary TJA were found to drop off significantly after a surgeon reaches a case volume of 35 per year [18]. It can be reasonably extrapolated that a similar association would be found between PJI case volume and outcome measurements. While the number of required revisions to define "highvolume" is currently unknown, ICM has set the recommendation for case volume to be a minimum of 25 based off this knowledge [17]. In addition to surgical technique, surgeons must be familiar with diagnostic and treatment algorithms that have been empirically proven on an international level to improve outcomes after PJI [3, 4].
Given the rarity of PJI cases, meeting the volume needed to gain expertise is difficult even at tertiary referral centers, let alone general hospitals. One strategy to address this and increase case volume for surgeons practicing in less densely populated settings may be to assign one or two surgeons with subspecialty interest and experience to handle PJI. While the literature on this strategy is fairly scarce, a study done by Matar et al. found that such specialists at a general hospital were able to produce comparable results when compared to a high-volume tertiary center if given an adequate caseload [16]. If this is not possible, PJI cases that present to general hospitals should strongly be considered for referral [12].

\section{Who Should Be Treated at a General Hospital? Who Should Be Referred?}

In an ideal world, there is only one circumstance in which patients should be treated at a community hospital by an unexperienced surgeon. The unstable patient, presenting with acute sepsis as a result of a PJI should be treated promptly prior to referral [12]. In this circumstance, catastrophe is imminent, and it is common sense that they cannot be transferred. However, it is recommended that all other cases be transferred prior to surgical management $[11,19]$. This includes patients who present in stable condition with acute post-operative, acute hematogenous, or chronic infection [12]. This recommendation was previously based on clinical judgment. However, it is now supported by the literature as well. Prior surgical intervention has been found to increase the risk of failure of subsequent surgical management of PJI $[12,19]$. The prevalence of culture-negative PJI was also found to be much higher when surgical intervention is attempted prior to referral to a tertiary care center, making management much more difficult [19]. Furthermore, patients with a history of prior treatment failure for PJI have been found to undergo less salvage procedures when treated at a high volume center as well, offering a possible prevention strategy for this circumstance [5, 6, 11].

Unfortunately, medicine can only be practiced within the constraints already set in place and while the evidence supports the creation of specialized tertiary centers and the use of a multidisciplinary approach as a long term solution, this may not be feasible under the current infrastructure [7]. In the meantime, patients should be risk-stratified in order to ensure that difficult cases are treated appropriately by orthopedic surgeons with the most experience in PJI. Patients at risk for infection after primary TJA are the same patients at high risk of recurrent infection and can be identified and referred even before infection takes place. Factors such as body mass index (BMI), malnutrition, Diabetes Mellitus (DM), Chronic Kidney Disease (CKD), smoking, and cardiovascular 
disease, among others, should all be taken into account. ${ }^{20}$ Continuity of care can be maintained if these patients are treated at specialized centers as well, which could help decrease the burden put on patients and their families.

If PJI has already been diagnosed, identifying which patients are at risk for failure of treatment and require referral also is clearer than ever. Kheir et al. developed a risk calculator for failure of treatment of PJI that may help in identifying such cases and found that, in descending order of importance; the need for irrigation and debridement, history of MI, revision surgery, presence of a sinus tract, culture of a resistant organism, smoking, history of prior surgery, synovial white blood cell count, body mass index, and erythrocyte sedimentation rate were all associated with an increased risk of treatment failure of PJI [13].

The superiority of treatment of complex cases by specialized centers is becoming evident as well. A study recently published by Ibrahim et al focused on the treatment of such cases, including patients with immunocompromise, systemic disease, concurrent sepsis, reinfection, and complex anatomy such as bone loss, significant soft-tissue compromise, and unidentified organisms, treated at a tertiary center with a high volume of PJI cases by multidisciplinary approach. The results of the study were that only 3 of 81 cases were reinfected. They reported a $90 \%$ eradication of MRSA infection with only 2 of 20 patients becoming reinfected, which is staggering compared to previous failure rates reported in the literature [11].

\section{Conclusion}

Periprosthetic Joint Infection is a complex disease state that requires constant communication and collaboration between a multidisciplinary team in order to provide the best treatment possible. Ideally, PJI cases should be treated by subspecialized surgeons who have experience treating a high volume of cases. Hospitals that aren't able to accommodate such patients should refer to centers that can, except in the unstable patient $[3,12,19]$. If this is not feasible, patients should be risk stratified, and transfer of the most complex cases to centers with experience in treating such cases is recommended. Standard protocols consistent with published guidelines to treat PJI should be established and adhered to at hospitals and, if possible, networks between general and tertiary centers should be established to provide support $[5,10,16]$.

\section{Authors' contribution}

S. Yacovelli - review of literature, writting the text. J. Parvizi - study concept, writting the text.

\section{References}

1. Kurtz S.M., Lau E., Schmier J., Ong K.L., Zhao K., Parvizi J. Infection burden for hip and knee arthroplasty in the United States. J Arthroplasty. 2008;23(7):984-991. doi: 10.1016/j.arth.2007.10.017.

2. Parvizi J., Tan T.L., Goswami K., Higuera C., Della Valle C., Chen A.F., Shohat N.The 2018Definition of Periprosthetic Hip and Knee Infection: An Evidence-Based and Validated Criteria. J Arthroplasty. 2018;33(5):1309-1314. e2. doi: 10.1016/j.arth.2018.02.078.

3. Tetreault M.W., Estrera K.A., Kayupov E., Brander C., Della Valle C.J. Are patients being evaluated for periprosthetic joint infection prior to referral to a tertiary care center? Arthroplasty Today. 2017;4(2): 216-220. doi: 10.1016/j.artd.2017.10.001.

4. Armstrong M.D., Carli A.V., Abdelbary H., Poitras S., Lapner P., Beaulé P.E. Tertiary care centre adherence to unified guidelines for management of periprosthetic joint infections: a gap analysis. Can J Surg. 2018;61(1): 34-41. doi:10.1503/cjs.008617.

5. Anderson M.B., Arciola C.R., Sarvanan S.A., Campoccia D., Certain L., Diaz-Ledezma C. et al. General Assembly, Treatment, Multidisciplinary Issues: Proceedings of International Consensus on Orthopedic Infections. J Arthroplasty. 2019;34(2S):S239-S243. doi: 10.1016/j.arth.2018.09.075.

6. Son M.-S., Lau E., Parvizi J., Mont M.A., Bozic K.J., Kurtz S. What Are the Frequency, Associated Factors, and Mortality of Amputation and Arthrodesis After a Failed Infected TKA? Clin Orthop Relat Res. 2017;475(12): 2905-2913. doi:10.1007/s11999-017-5285

7. Dietz M.J., Springer B.D., Barnes P.D., Falciglia M., Friedrich A.D., Berendt A.R. et al. Best practices for centers of excellence in addressing periprosthetic joint infection. J Am Acad Orthop Surg. 2015;23 Suppl:S12-7. doi: 10.5435/JAAOS-D-14-00380.

8. Ntalos D., Berger-Groch J., Rohde H., Grossterlinden L.G., Both A., Luebke A. et al. Implementation of a multidisciplinary infections conference affects the treatment plan in prosthetic joint infections of the hip: a retrospective study. Arch Orthop Trauma Surg. 2019;139(4):467-473. doi: 10.1007/s00402-018-3079-6.

9. Yan C.H., Arciola C.R., Soriano A., Levin L.S., Bauer T.W., Parvizi J. Team Approach: The Management of Infection After Total Knee Replacement. JBJS Rev. 2018;6(4):e9. doi: 10.2106/JBJS.RVW.17.00058.

10. Minassian A.M., Osmon D.R., Berendt A.R. Clinical guidelines in the management of prosthetic joint infection. J Antimicrob Chemother. 2014;69 Suppl 1:i2935.doi: 10.1093/jac/dku253.

11. Ibrahim M.S., Raja S., Khan M.A., Haddad F.S. A multidisciplinary team approach to two-stage revision for the infected hip replacement: a minimum five-year follow-up study. Bone Joint J. 2014;96-B(10):1312-318. doi: 10.1302/0301-620X.96B10.32875.

12. Petretta R., Phillips J., Toms A. Management of acute periprosthetic joint infection of the knee - Algorithms for the on call surgeon. Surgeon. 2017;15(2):83-92. doi:10.1016/j.surge.2016.06.001.

13. Kheir M.M., Tan T.L., George J., Higuera C.A., Maltenfort M.G., Parvizi J. Development and Evaluation of a Prognostic Calculator for the Surgical Treatment of Periprosthetic Joint Infection. J Arthroplasty. 2018;33(9):2986-2992.e1. doi: 10.1016/j.arth.2018.04.034. 
14. Jayakumar P., Moore M.L.G., Bozic K.J. Team Approach: A Multidisciplinary Approach to the Management of Hip and Knee Osteoarthritis. JBJS Rev. 2019;7(6):e10. doi: 10.2106/jbjs.rvw.18.00133.

15. Akgün D., Müller M., Perka C., Winkler T. High cure rate of periprosthetic hip joint infection with multidisciplinary team approach using standardized two-stage exchange. J Orthop Surg Res. 2019;14(1):78. doi: 10.1186/s13018-019-1122-0.

16. Matar H.E., Stritch P., Emms N. Two-stage revisions of infected hip replacements: Subspecialisation and patient-reported outcome measures. J Orthop. 2019;16(2):179-181. doi: 10.1016/j.jor.2019.02.011.

17. Bravo T., Budhiparama N., Flynn S., Gaol I.L., Hidayat H., Ifran N.N. et al. Hip and Knee Section, Prevention, Postoperative Issues: Proceedings of International
Consensus on Orthopedic Infections. J Arthroplasty. 2019;34(2S):S321-S323. doi: 10.1016/j.arth.2018.09.017.

18. Ravi B., Jenkinson R., Austin P.C., Croxford R., Wasserstein D., Escott B. et al. Relation between surgeon volume and risk of complications after total hip arthroplasty: propensity score matched cohort study. BMJ. 2014;348:g3284. doi: 10.1136/bmj.g3284.

19. Song S.Y., Goodman S.B., Suh G., Finlay A.K., Huddleston J.I. 3rd, Maloney W.J., Amanatullah D.F. Surgery Before Subspecialty Referral for Periprosthetic Knee Infection Reduces the Likelihood of Infection Control. Clin Orthop Relat Res. 2018;476(10):1995-2002. doi: 10.1097/corr.0000000000000423.

20. Parvizi J., Shohat N., Gehrke T. Prevention of periprosthetic joint infection. Bone Joint J. 2017;99-B(4_Supple_B):3-10. doi: 10.1302/0301-620x.99b4.bjj-2016-1212.r1.

\section{AUTHORS' AFFILATIONS:}

Steven Yacovelli - MD, Researcher Fellow, Rothman Orthopedic Institute, Department of Orthopedics, Thomas Jefferson University Hospital, Philadelphia, USA

Javad Parvizi - MD, Professor, Director of Clinical Research, Rothman Orthopedic Institute, Department of Orthopedics Thomas Jefferson University Hospital, Philadelphia, USA 